\title{
Self-assembly and Formation of Chromonic \\ Liquid Crystals from the Dyes Quinaldine Red
}

\section{Acetate and Pyronin Y}

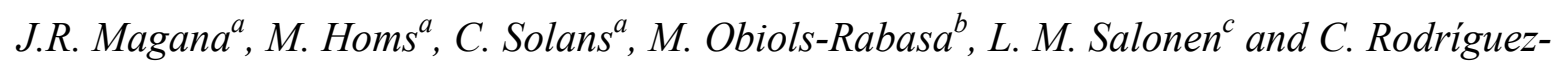
Abreu $^{c, *}$

${ }^{a}$ Instituto de Química Avanzada de Cataluña, Consejo Superior de Investigaciones Científicas (IQAC-CSIC), CIBER de Bioingeniería, Biomateriales y Nanomedicina (CIBER-BBN), Jordi Girona 18-26, 08034 Barcelona, Spain.

${ }^{b}$ Division of Physical Chemistry, Lund University, Getingevägen 60, SE-22241 Lund, Sweden.

${ }^{c}$ International Iberian Nanotechnology Laboratory, Av. Mestre José Veiga s/n, 4715330 Braga, Portugal. 


\section{ASSOCIATED CONTENT}

\section{Supporting information}

\section{NMR Spectroscopy}

Proton chemical shifts are expressed in parts per million ( $\delta$ scale) and are calibrated using the residual undeuterated solvent peak as an internal reference $\left(\mathrm{D}_{2} \mathrm{O}: \delta 4.80 ;\left(\mathrm{CD}_{3}\right)_{2} \mathrm{SO}\right.$ : $\delta$ 2.50). Data for ${ }^{1} \mathrm{H}$ NMR spectra are reported as follows: chemical shift $(\delta, \mathrm{ppm})$ (multiplicity, coupling constant/Hz, integration, assignment). Multiplicities are reported as follows: $\mathrm{s}=$ singlet, $\mathrm{d}=$ doublet, $\mathrm{t}=$ triplet, $\mathrm{q}=$ quartet, $\mathrm{m}=$ multiplet, or combinations thereof. Carbon chemical shifts are expressed in ppm ( $\delta$ scale) and are referenced to the carbon resonances of the solvent $\left.\left(\mathrm{CD}_{3}\right)_{2} \mathrm{SO}: \delta 39.52\right)$.

Atom numbering is arbitrary. The assignment of protons was done based on twodimensional NMR spectroscopy experiments. 


\section{Quinaldine Red Acetate}

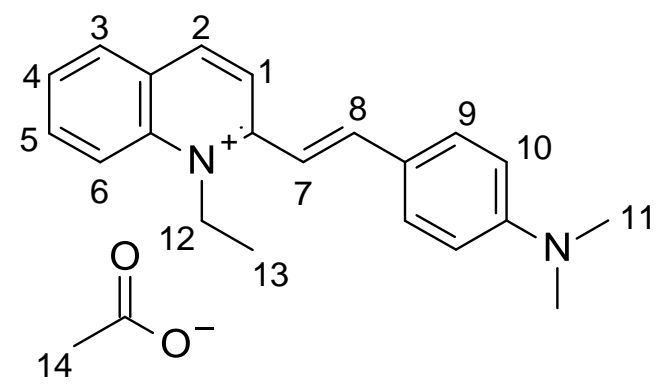

${ }^{1} \mathrm{H}$ NMR (400 MHz, D $\left.2 \mathrm{O}\right): 1.40$ (t, $\left.J=7.4 \mathrm{~Hz}, 3 \mathrm{H}, \mathrm{H}-\mathrm{C}(13)\right), 1.93$ (s, $\left.3 \mathrm{H}, \mathrm{H}-\mathrm{C}(14)\right)$, 2.35 (s, $6 \mathrm{H}, \mathrm{H}-\mathrm{C}(11)), 4.27$ (q, $J=8.0 \mathrm{~Hz}, 2 \mathrm{H}, \mathrm{H}-\mathrm{C}(12)), 5.73$ (d, J=8.80 Hz, $2 \mathrm{H}, \mathrm{H}-$ $\mathrm{C}(10)), 6.43(\mathrm{~d}, J=15.6 \mathrm{~Hz}, 1 \mathrm{H}, \mathrm{H}-\mathrm{C}(7)), 6.87$ (d, $J=8.8 \mathrm{~Hz}, 2 \mathrm{H}, \mathrm{H}-\mathrm{C}(9)), 7.33$ (d, $J=$ 15.2 Hz, $1 \mathrm{H}, \mathrm{H}-\mathrm{C}(8)), 7.51-7.55$ (m, $1 \mathrm{H}, \mathrm{H}-\mathrm{C}(4)), 7.62$ (d, $J=9.6 \mathrm{~Hz}, 1 \mathrm{H}, \mathrm{H}-\mathrm{C}(1))$, $7.70(\mathrm{~d}, J=8.0 \mathrm{~Hz}, 1 \mathrm{H}, \mathrm{H}-\mathrm{C}(3)), 7.73-7.79(\mathrm{~m}, 2 \mathrm{H}, \mathrm{H}-\mathrm{C}(5)$ and $\mathrm{H}-\mathrm{C}(6)), 8.00(\mathrm{~d}, J=8.8$ $\mathrm{Hz}, 1 \mathrm{H}, \mathrm{H}-\mathrm{C}(2)) ;{ }^{13} \mathrm{C} \mathrm{NMR}\left(100 \mathrm{MHz}, \mathrm{D}_{2} \mathrm{O}\right): 12.9,23.1,38.4,45.5,108.0,110.7,116.9$, $118.4,120.4,126.6,127.4,129.5,130.7,133.8,137.4,141.2,147.9,151.6,153.4,181.2$.

${ }^{1} \mathrm{H}$ NMR (400 MHz, $\left.\left(\mathrm{CD}_{3}\right)_{2} \mathrm{SO}\right): 1.54(\mathrm{t}, J=7.1 \mathrm{~Hz}, 3 \mathrm{H}, \mathrm{H}-\mathrm{C}(13)), 1.55$ (s, $3 \mathrm{H}, \mathrm{H}-$ C(14)), 3.09 (s, $6 \mathrm{H}, \mathrm{H}-\mathrm{C}(11)), 5.05$ (q, $J=7.1 \mathrm{~Hz}, 2 \mathrm{H}, \mathrm{H}-\mathrm{C}(12)), 6.84$ (d, J = 9.0 Hz, 2 $\mathrm{H}, \mathrm{H}-\mathrm{C}(10)), 7.47(\mathrm{~d}, J=15.4 \mathrm{~Hz}, 1 \mathrm{H}, \mathrm{H}-\mathrm{C}(7)), 7.82-7.89(\mathrm{~m}, 3 \mathrm{H}, \mathrm{H}-\mathrm{C}(9)$ and $\mathrm{H}-\mathrm{C}(4))$, $8.09(\mathrm{ddd}, J=8.8,7.2,1.6 \mathrm{~Hz}, 1 \mathrm{H}, \mathrm{H}-\mathrm{C}(5)), 8.25$ (dd, $J=8.0,1.4 \mathrm{~Hz}, 1 \mathrm{H}, \mathrm{H}-\mathrm{C}(3)), 8.32$ $(\mathrm{d}, J=15.4 \mathrm{~Hz}, 1 \mathrm{H}, \mathrm{H}-\mathrm{C}(8)), 8.44(\mathrm{~d}, J=9.0 \mathrm{~Hz}, 1 \mathrm{H}, \mathrm{H}-\mathrm{C}(6)), 8.54(\mathrm{~d}, J=9.3 \mathrm{~Hz}, 1 \mathrm{H}$, $\mathrm{H}-\mathrm{C}(1)), 8.80(\mathrm{~d}, J=9.2 \mathrm{~Hz}, 1 \mathrm{H}, \mathrm{H}-\mathrm{C}(2)) ;{ }^{13} \mathrm{C}$ NMR $\left(100 \mathrm{MHz},\left(\mathrm{CD}_{3}\right)_{2} \mathrm{SO}\right): 13.8,24.8$, $45.5,110.7,111.9,118.4,120.3,122.4,127.1,128.0,130.1,132.1,134.4,138.1,142.0$ 149.7, 153.0, 155.4, 172.6 (one signal hidden under the carbon resonance of the solvent). 
H NMR (400 MHz, D 20 ), QR-Ac 0.5 wt $\%$

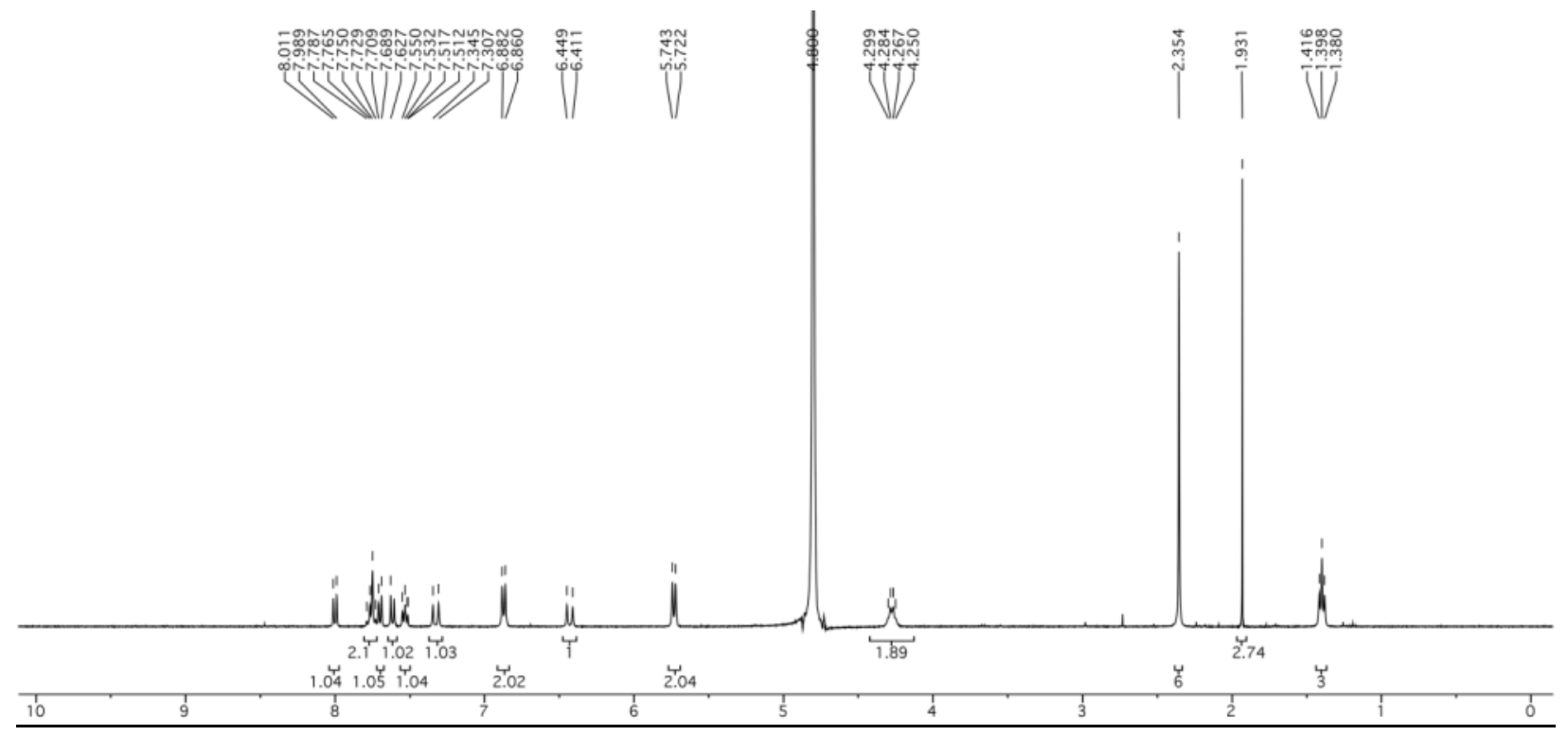


${ }^{13} \mathrm{C}$ NMR (100 MHz, D $\left.2 \mathrm{O}\right)$, QR-Ac 0.5 wt $\%$
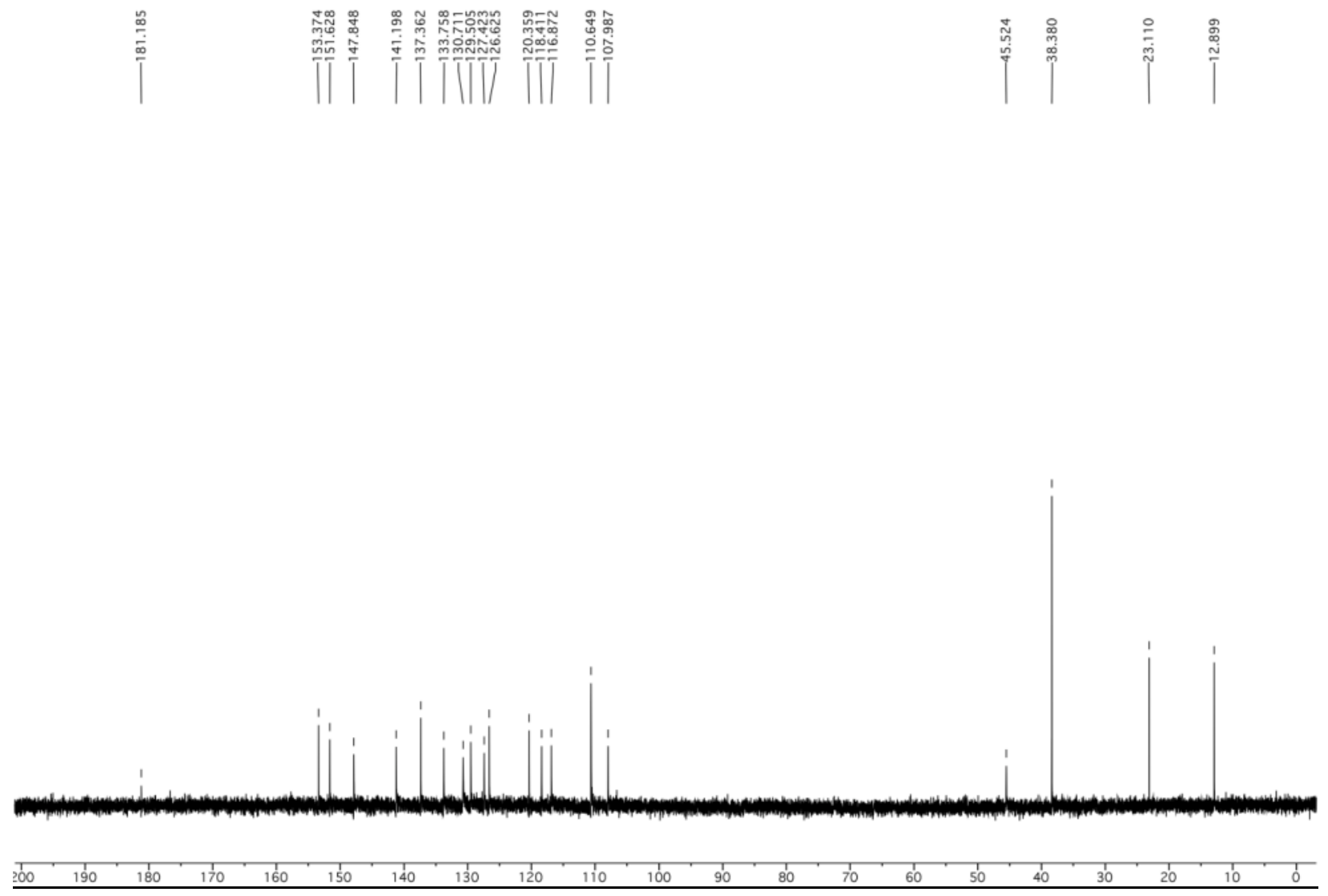
${ }^{1} \mathrm{H}$ NMR (400 MHz, $\left.\left(\mathrm{CD}_{3}\right)_{2} \mathrm{SO}\right)$, QR-Ac $0.5 \mathrm{wt} \%$

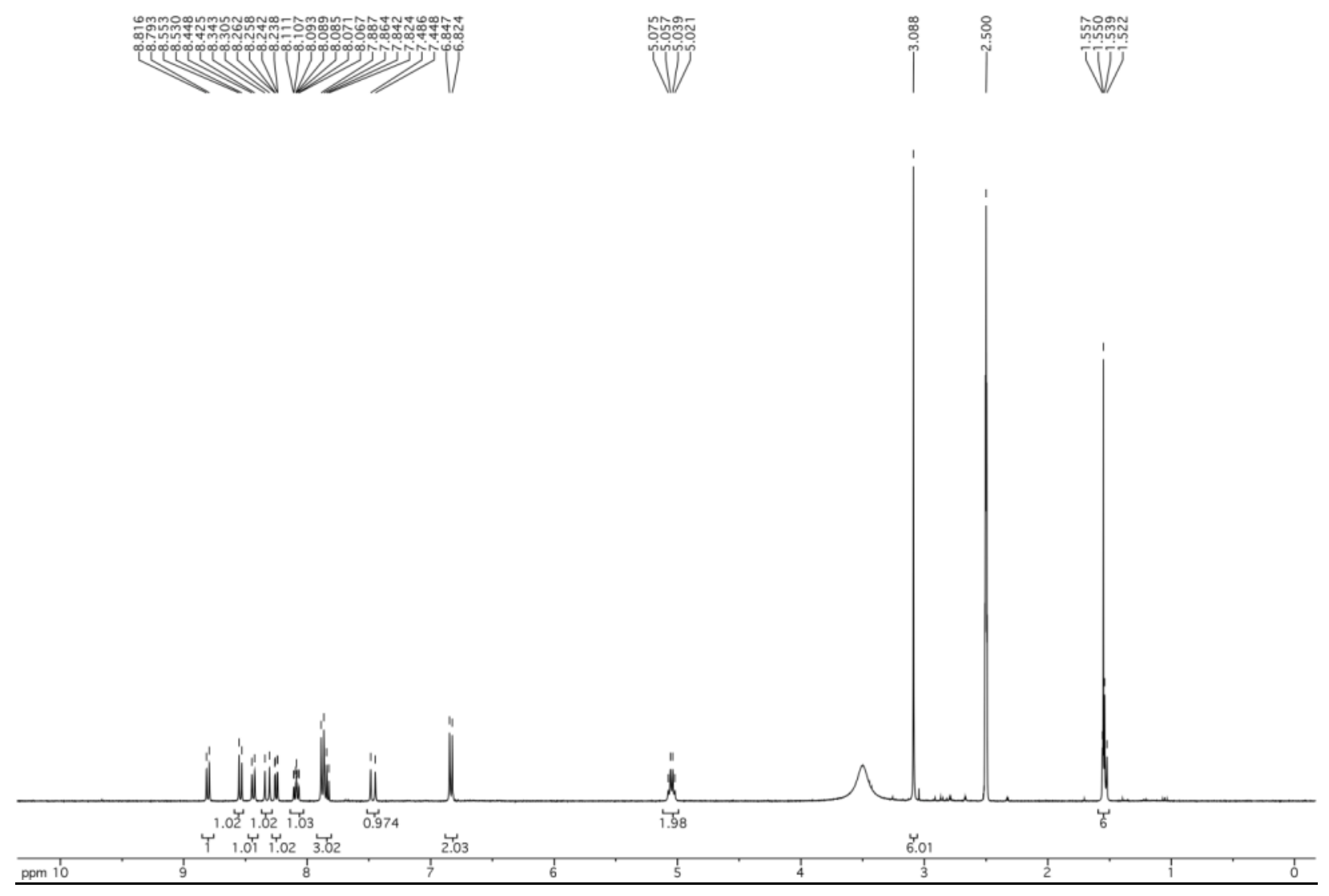


${ }^{13} \mathrm{C}$ NMR (100 MHz, (CD3)2SO), QR-Ac $0.5 \mathrm{wt} \%$
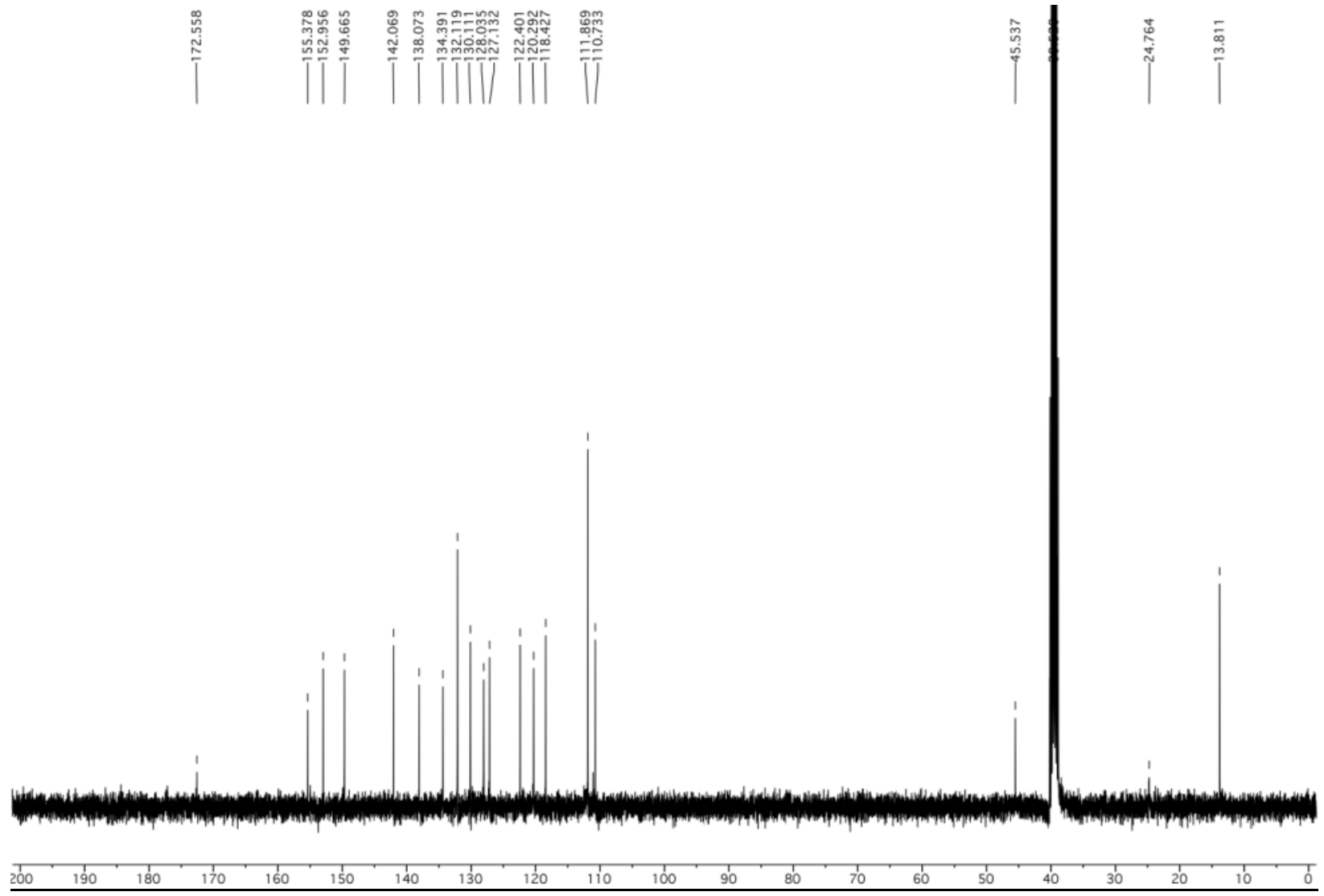
2D ${ }^{1} \mathrm{H}-{ }^{1} \mathrm{H}$ NOESY (400 MHz, $\mathrm{D}_{2} \mathrm{O}$ ); QR-Ac $0.5 \mathrm{wt} \%$

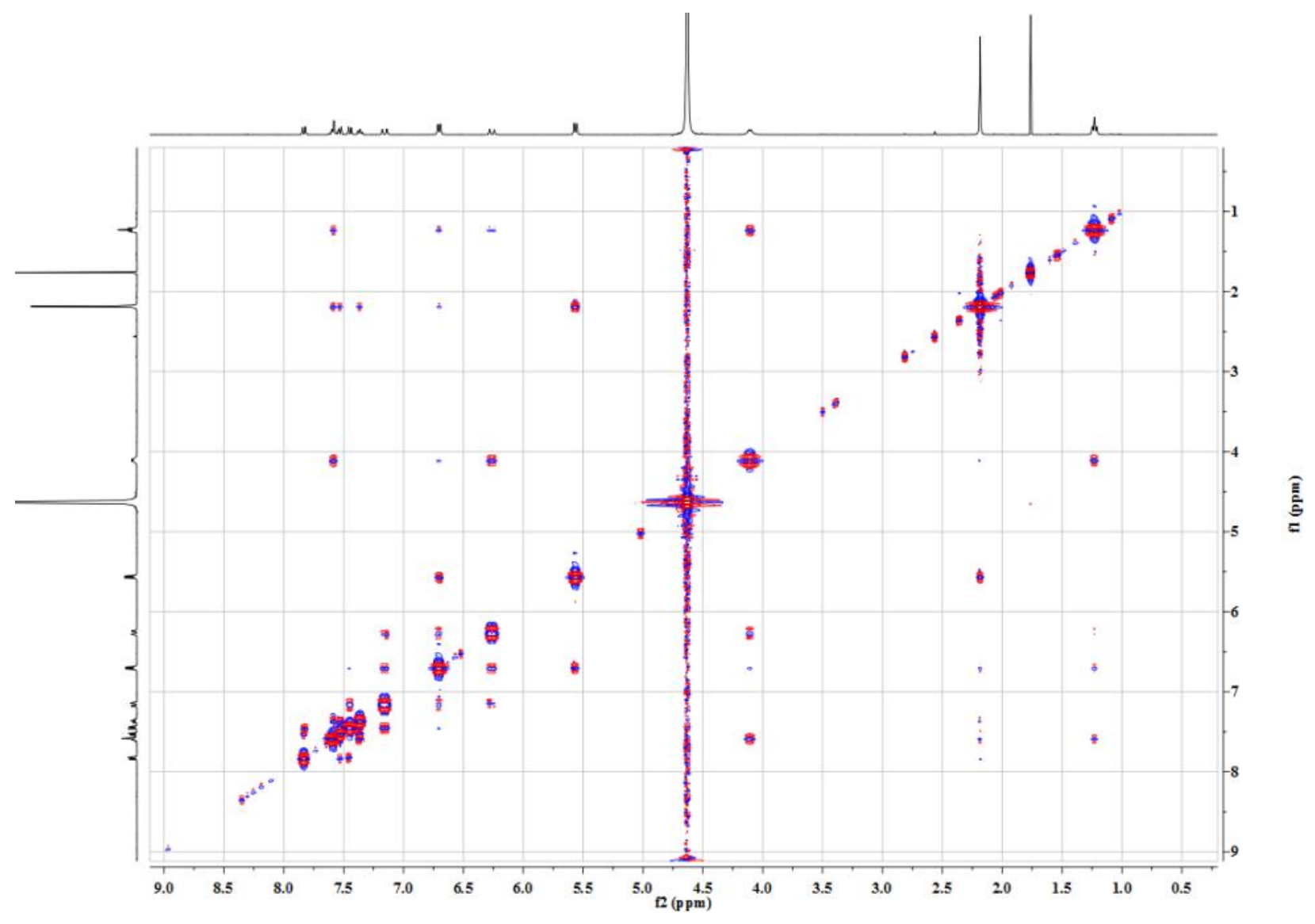


2D ${ }^{1} \mathrm{H}-{ }^{1} \mathrm{H}$ NOESY (400 MHz, $\left.\left(\mathrm{CD}_{3}\right)_{2} \mathrm{SO}\right)$; QR-Ac $0.5 \mathrm{wt} \%$

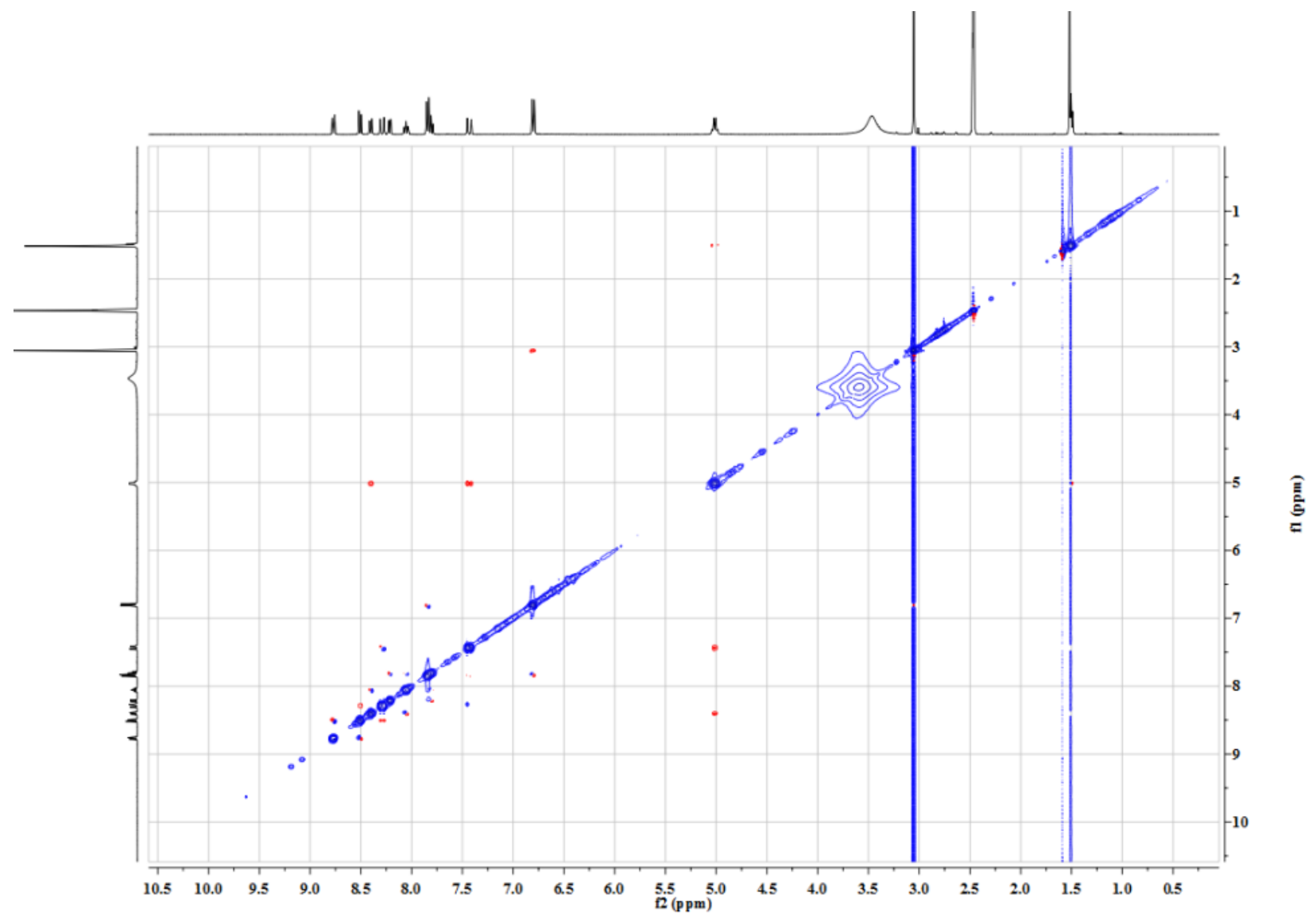




\section{Pyronin Y}

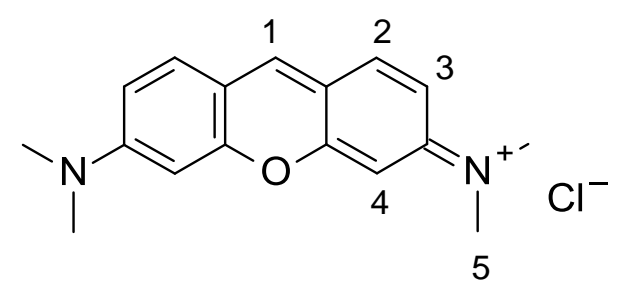

${ }^{1} \mathrm{H}$ NMR (400 MHz, D $2 \mathrm{O}$ ): 3.03 (s, 12 H, H-C(5)), 6.16 (s, $\left.2 \mathrm{H}, \mathrm{H}-\mathrm{C}(4)\right), 6.67$ (dd, $J=$ 9.1, 1.6 Hz, $3 \mathrm{H}, \mathrm{H}-\mathrm{C}(3)), 7.23(\mathrm{~d}, J=9.2 \mathrm{~Hz}, 2 \mathrm{H}, \mathrm{H}-\mathrm{C}(2)), 7.82$ (s, $1 \mathrm{H}, \mathrm{H}-\mathrm{C}(1))$.

${ }^{1} \mathrm{H}$ NMR (500 MHz, D 20$)$, PyY 0.5 wt\%

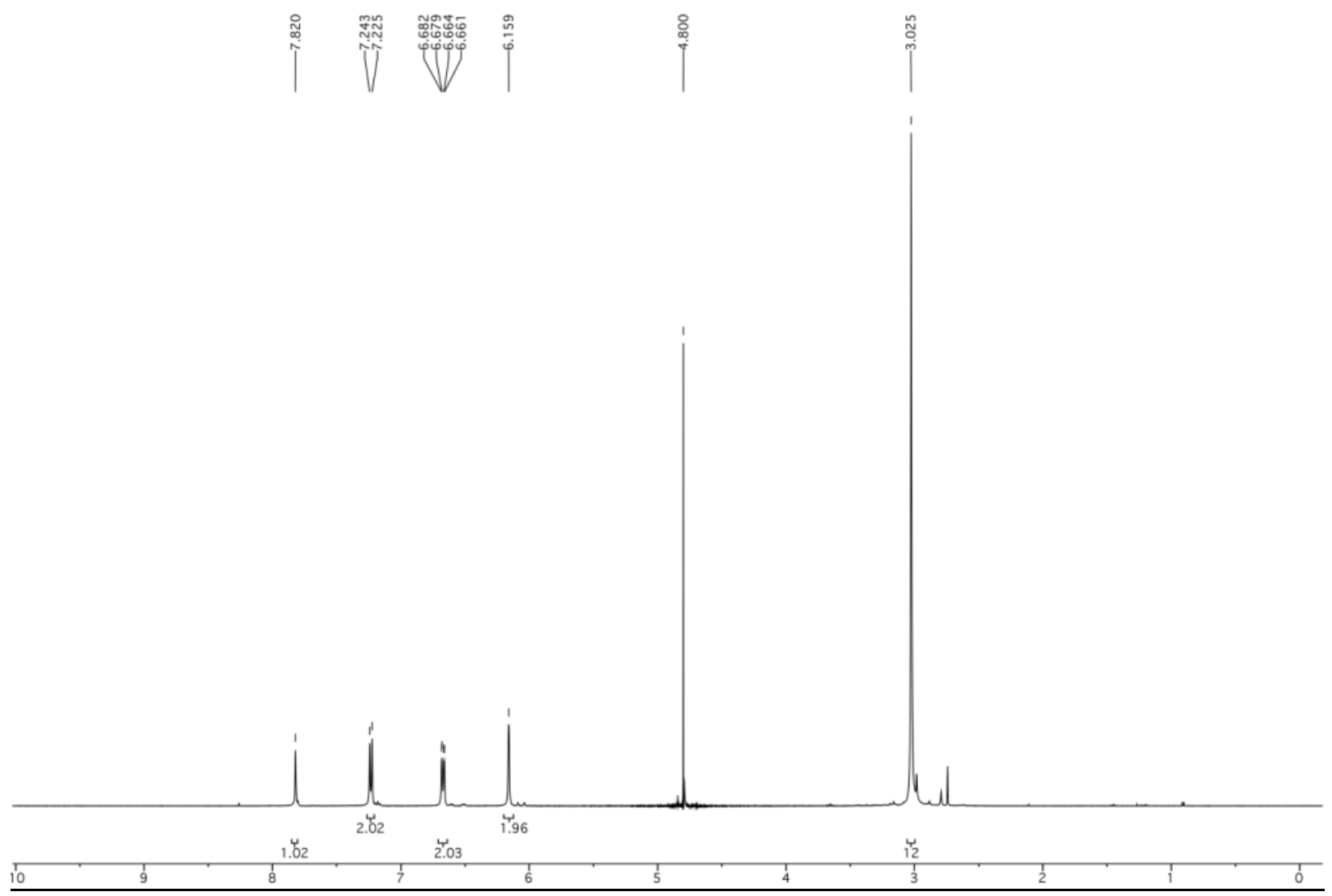


UV-Vis Spectroscopy. Measurements were performed at $25^{\circ} \mathrm{C}$ in a Varian Cary 300 instrument.

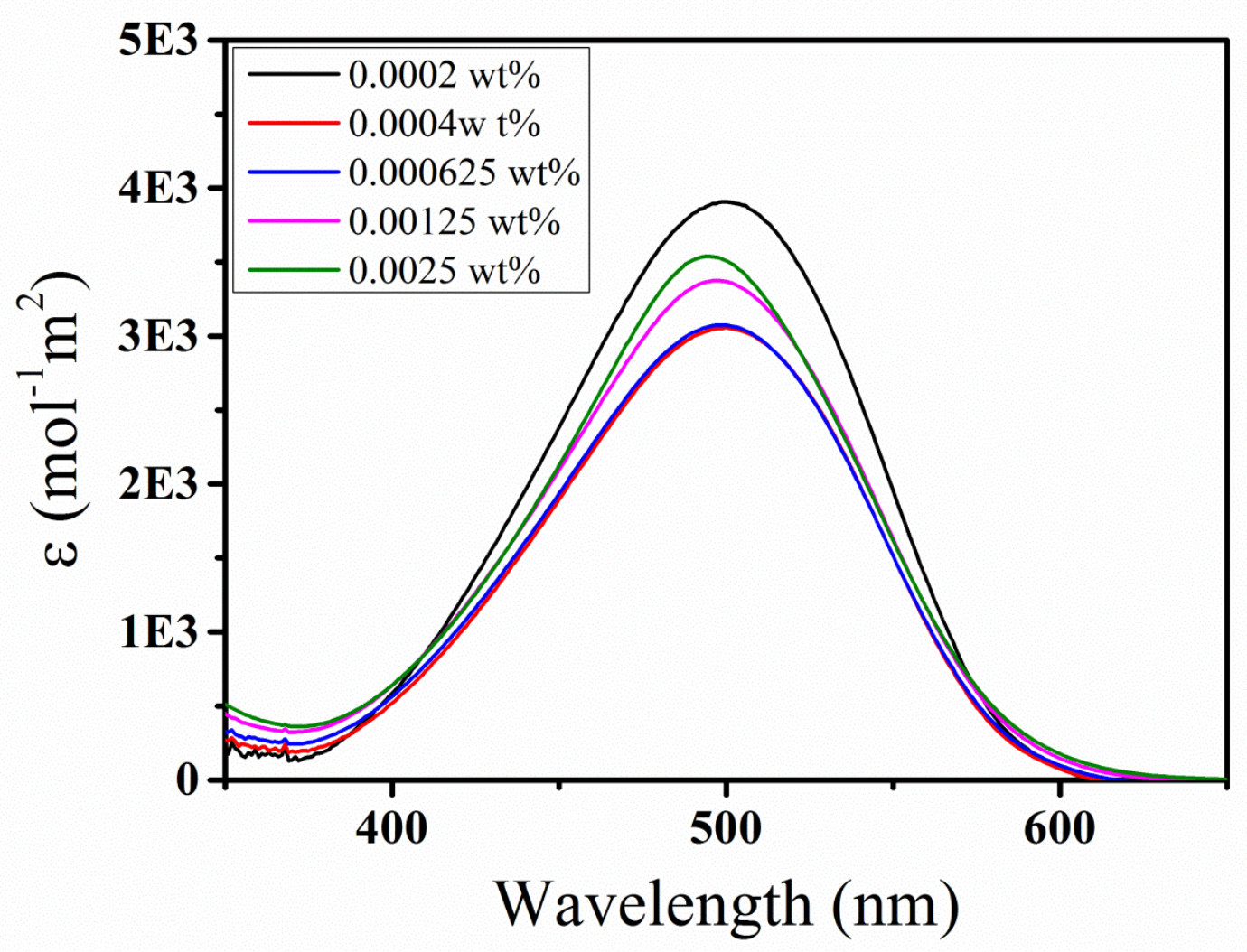

Figure SI1. Absorption spectra of QR-Ac at different concentrations in water at $25^{\circ} \mathrm{C}$. 
Fluorescence Spectroscopy. Measurements were performed at $25^{\circ} \mathrm{C}$ and at an excitation wavelength of $530 \mathrm{~nm}$ in a Varian Cary Eclipse 4000 instrument.

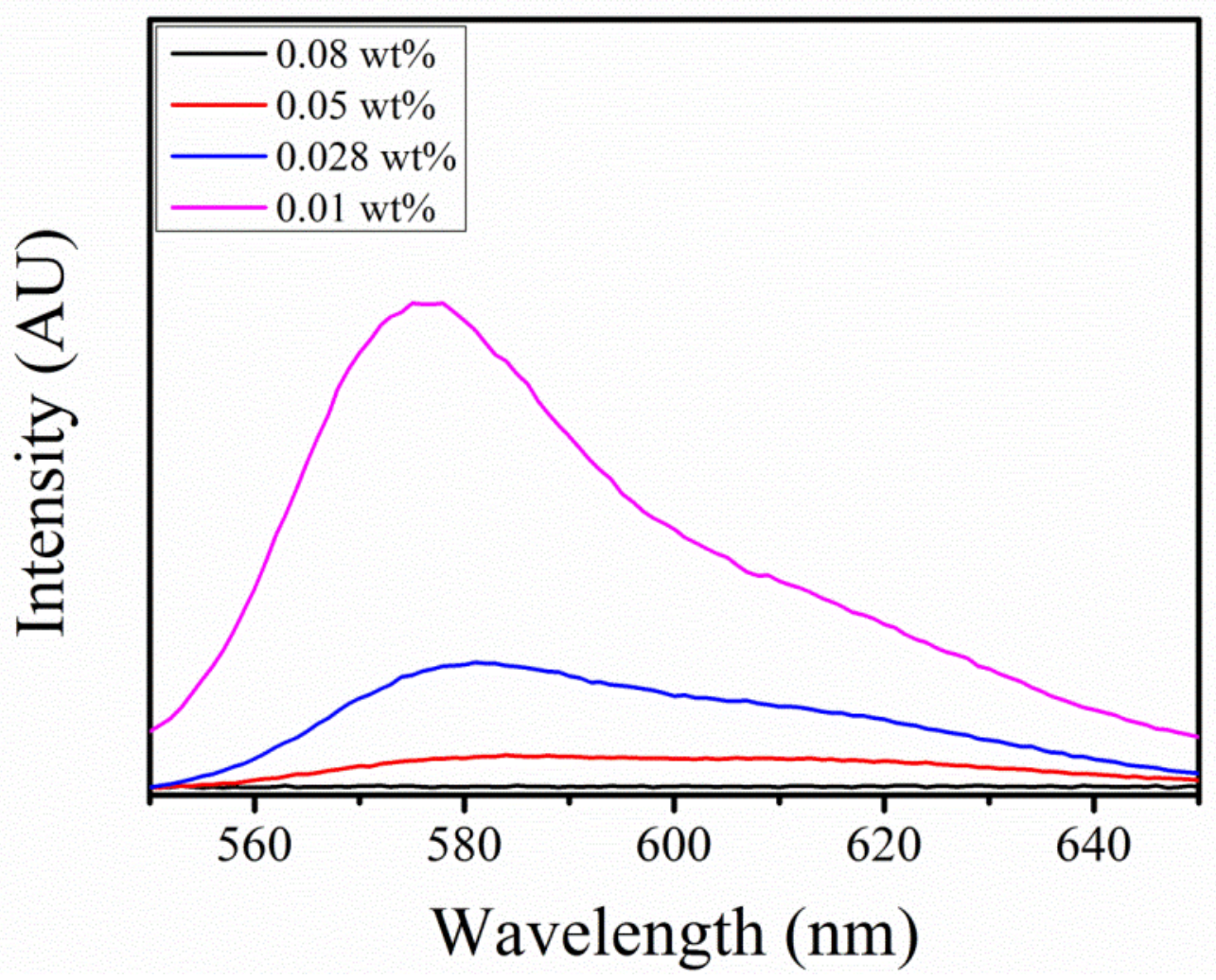

Figure SI2. Fluorescence spectra of PyY (excitation wavelength $=530 \mathrm{~nm}$ ) at different concentrations in water at $25^{\circ} \mathrm{C}$. The fluorescence is completely quenched at $0.08 \mathrm{wt} \%$. 
Micro-Differential Scanning Calorimetry (Micro-DSC). Samples were placed in a hermetically sealed $850 \mu 1$ hasteloid batch vessel. Measurements were recorded using a Setaram Micro-DSC III.

Micro-DSC scans for concentrated ( $>60 \mathrm{wt} \%)$ samples presented a small transition peak with enthalpy values of around $0.1 \mathrm{~kJ} / \mathrm{mol}$ (Figure SI3) that could be ascribed to a rectangular to lamellar phase transition. A larger peak at lower temperatures attributed to a crystalline solid-liquid crystal transition was also observed with an enthalpy value of around $3 \mathrm{~kJ} / \mathrm{mol}$.

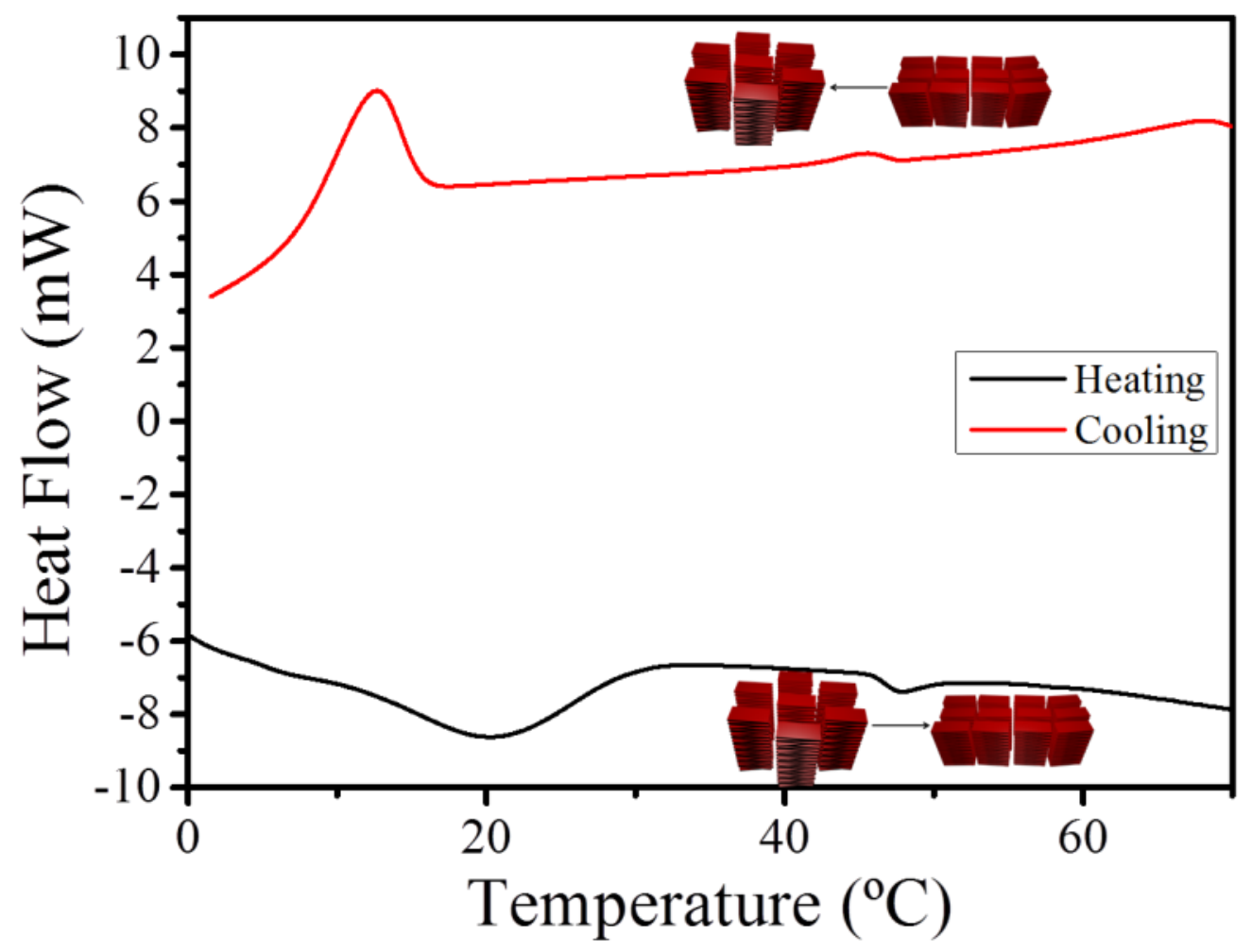

Figure SI3. Micro DSC curves of a $68 \mathrm{wt} \%$ QR-Ac sample in water. 
Small and wide angle $\mathbf{X}$-ray Scattering. Measurements were performed in an Anton Paar SAXSess $\mathrm{mc}^{2}$ instrument. Data were collected with an image plate detector. Samples were placed in flamne-sealed capillaries $(0.9 \mathrm{~mm}$ in diameter and $0.01 \mathrm{~mm}$ wall thickness) for the measurement.

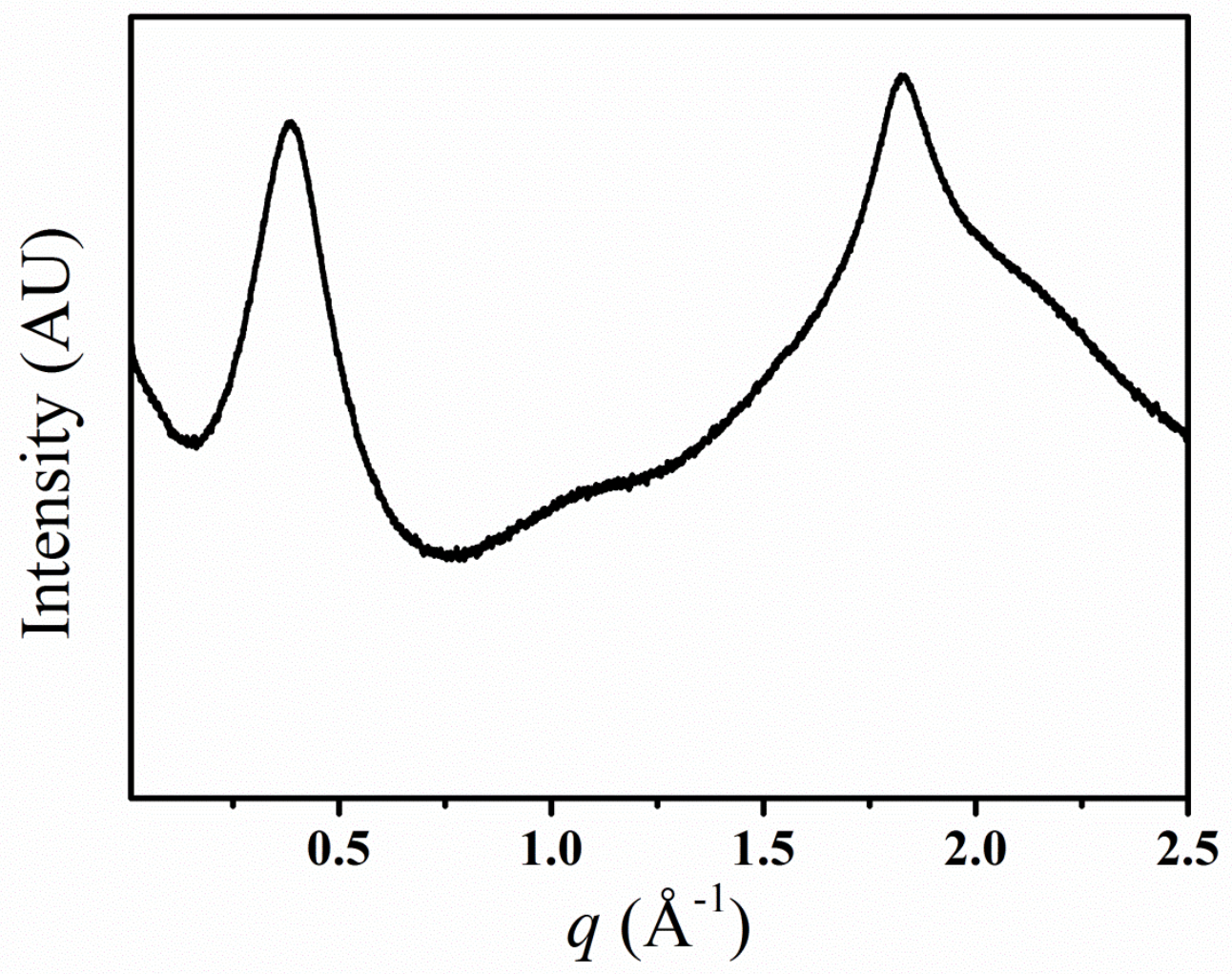

Figure SI4. Representative SAXS/WAXS pattern for a $50 \mathrm{wt} \% \mathbf{P y Y}$ sample at $30{ }^{\circ} \mathrm{C}$ in water. 


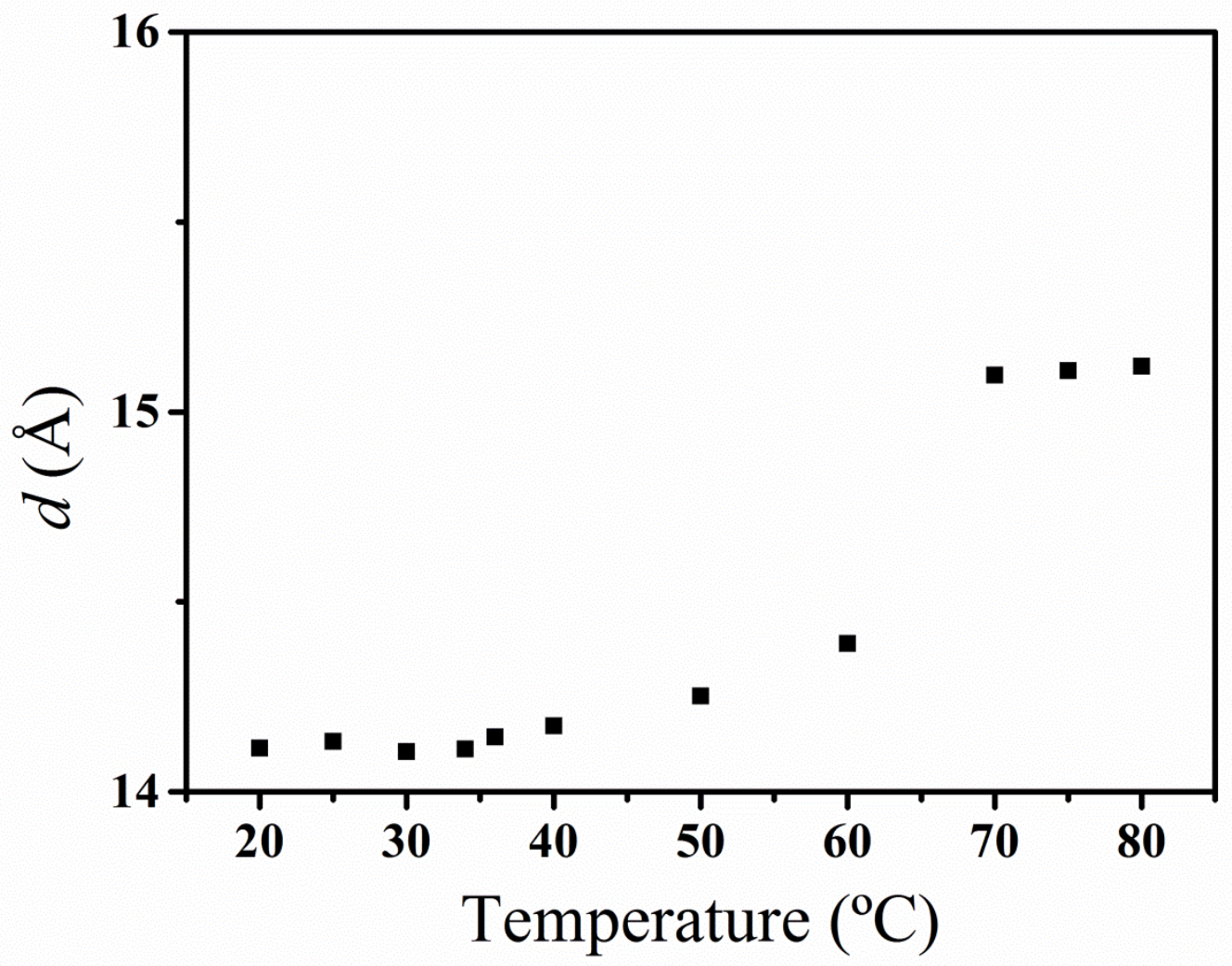

Figure SI5. Bragg spacing from the most intense peak in the dye nematic phase as a function of temperature for a $60 \mathrm{wt} \%$ PyY sample in water 


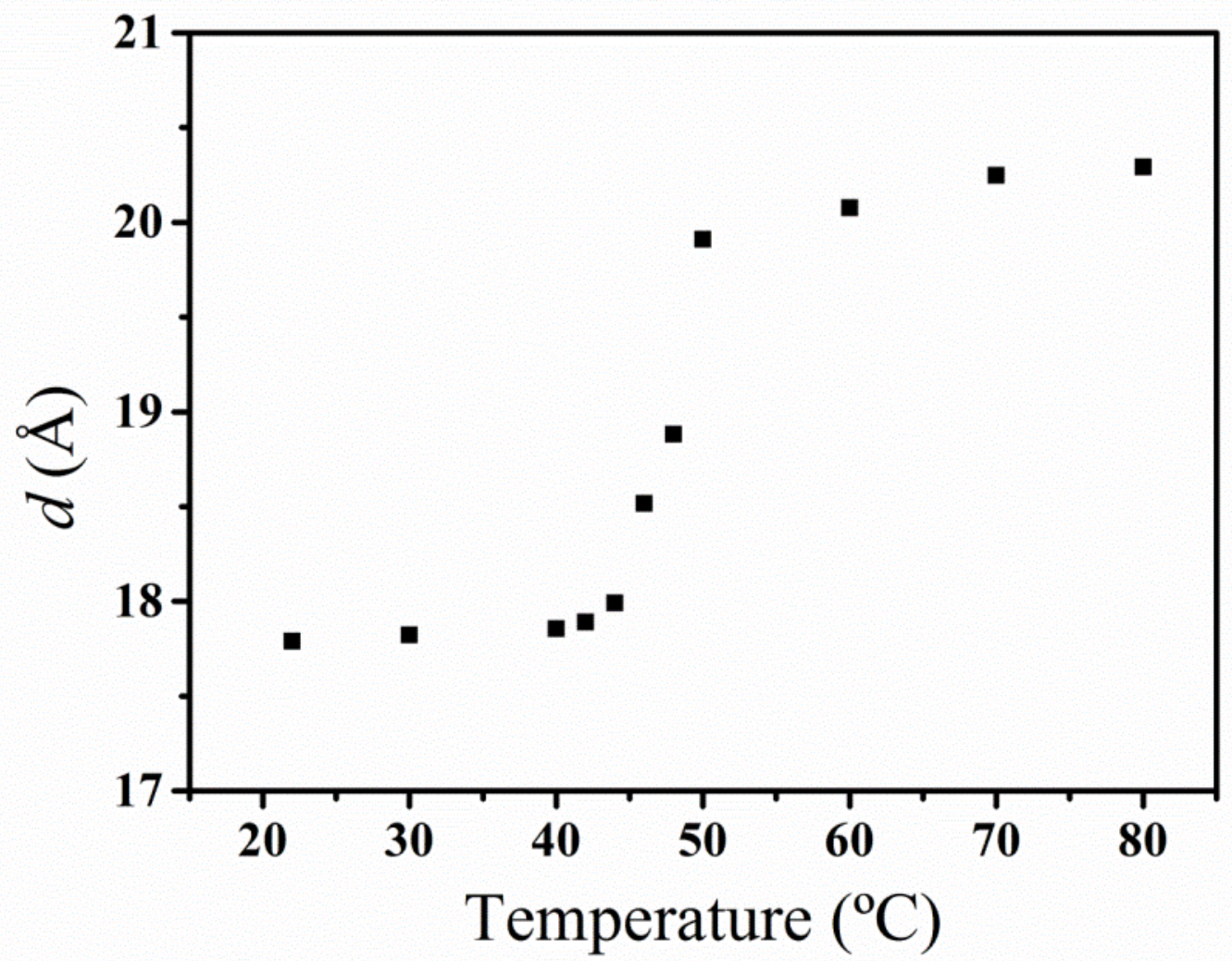

Figure SI6. Bragg spacing from the most intense peak in the dye nematic phase as a function of temperature for a $50 \mathrm{wt} \%$ QR-Ac sample in water. 
Table SI1. Experimental SAXS peak positions for the QR-Ac/water O phase at different concentrations $\left(\right.$ Temperature $\left.=30^{\circ} \mathrm{C}\right)$

\begin{tabular}{|c|c|c|c|c|}
\hline $\begin{array}{c}\text { Concentration } \\
(\mathbf{w t \%})\end{array}$ & $\begin{array}{c}\boldsymbol{q}_{\mathbf{0 2}} \\
(\mathbf{\AA})\end{array}$ & $\begin{array}{c}\boldsymbol{q}_{\mathbf{1 1}} \\
(\mathbf{\AA})\end{array}$ & $\begin{array}{c}\boldsymbol{q}_{\mathbf{0}} \\
(\mathbf{\AA})\end{array}$ & $\begin{array}{c}\boldsymbol{q}_{\mathbf{2}} \\
(\AA)\end{array}$ \\
\hline 59.37 & 0.369 & 0.450 & -- & -- \\
\hline 60.25 & 0.375 & 0.458 & -- & -- \\
\hline 61.75 & 0.379 & 0.465 & 0.760 & 0.928 \\
\hline 64.4 & 0.388 & 0.479 & 0.773 & 0.946 \\
\hline 66 & 0.401 & 0.495 & 0.808 & 0.987 \\
\hline 68 & 0.407 & 0.500 & 0.818 & 1.001 \\
\hline
\end{tabular}

Table SI2. Experimental SAXS peak positions for the QR-Ac/water O phase at different concentrations $\left(\right.$ Temperature $\left.=35^{\circ} \mathrm{C}\right)$

\begin{tabular}{|c|c|c|c|c|}
\hline $\begin{array}{c}\text { Concentration } \\
(\mathbf{w t} \%)\end{array}$ & $\begin{array}{c}\boldsymbol{q}_{\mathbf{0 2}} \\
(\mathbf{\AA})\end{array}$ & $\begin{array}{c}\boldsymbol{q}_{\mathbf{1 1}} \\
(\mathbf{\AA})\end{array}$ & $\begin{array}{c}\boldsymbol{q}_{\mathbf{0 4}} \\
(\AA)\end{array}$ & $\begin{array}{c}\boldsymbol{q}_{22} \\
(\AA)\end{array}$ \\
\hline 59.37 & -- & -- & -- & -- \\
\hline 60.25 & 0.373 & 0.454 & -- & -- \\
\hline 61.75 & 0.373 & 0.459 & 0.751 & 0.928 \\
\hline 64.4 & 0.383 & 0.469 & 0.768 & 0.937 \\
\hline 66 & 0.395 & 0.488 & 0.800 & 0.982 \\
\hline 68 & 0.401 & 0.498 & 0.810 & 0.992 \\
\hline
\end{tabular}

